\title{
Quantitative breast density analysis to predict interval and node-positive cancers in pursuit of improved screening protocols: a case-control study
}

\author{
Elizabeth S. Burnside ${ }^{1 凶}{ }^{凶}$, Lucy M. Warren ${ }^{2}$, Jonathan Myles ${ }^{3}$, Louise S. Wilkinson ${ }^{4}$, Matthew G. Wallis ${ }^{5}$, Mishal Patel ${ }^{6}$,
} Robert A. Smith (D) ${ }^{7}$, Kenneth C. Young ${ }^{2}$, Nathalie J. Massat $\mathbb{D}^{3}$ and Stephen W. Duffy $\mathbb{D}^{3}$

(c) The Author(s) 2021

\begin{abstract}
BACKGROUND: This study investigates whether quantitative breast density (BD) serves as an imaging biomarker for more intensive breast cancer screening by predicting interval, and node-positive cancers.

METHODS: This case-control study of 1204 women aged 47-73 includes 599 cancer cases (302 screen-detected, 297 interval; 239 node-positive, 360 node-negative) and 605 controls. Automated BD software calculated fibroglandular volume (FGV), volumetric breast density (VBD) and density grade (DG). A radiologist assessed BD using a visual analogue scale (VAS) from 0 to 100. Logistic regression and area under the receiver operating characteristic curves (AUC) determined whether BD could predict mode of detection (screen-detected or interval); node-negative cancers; node-positive cancers, and all cancers vs. controls.

RESULTS: FGV, VBD, VAS, and DG all discriminated interval cancers (all $p<0.01$ ) from controls. Only FGV-quartile discriminated screen-detected cancers $(p<0.01)$. Based on AUC, FGV discriminated all cancer types better than VBD or VAS. FGV showed a significantly greater discrimination of interval cancers, AUC $=0.65$, than of screen-detected cancers, AUC $=0.61$ ( $p<0.01)$ as did $\operatorname{VBD}(0.63$ and 0.53 , respectively, $p<0.001)$.

CONCLUSION: FGV, VBD, VAS and DG discriminate interval cancers from controls, reflecting some masking risk. Only FGV discriminates screen-detected cancers perhaps adding a unique component of breast cancer risk.
\end{abstract}

British Journal of Cancer (2021) 125:884-892; https://doi.org/10.1038/s41416-021-01466-y

\section{BACKGROUND}

The aim of stratified, or risk-based, breast cancer screening [1-5] is to optimise the balance of benefits of early cancer detection and mortality reduction with the harms of false-positive mammograms, benign biopsies, and overdiagnosis [4]. However, riskbased protocols may eliminate screening benefits for some women $[6,7]$ and increase complexity, with questionnaires, blood draws, and counselling, thereby potentially detracting from the performance of an age-based screening programme. To preserve or improve effectiveness, a stratified screening programme needs to maintain or decrease the incidence of advanced (i.e. nodepositive) and interval cancers, those tumours most likely to be clinically significant. Ideally, stratified protocols would personalise mammography initiation, screening interval, and supplemental screening with other modalities in order to decrease advanced and interval cancers, while maintaining low rates of false positives. This programme would ideally decrease mortality from breast cancer in all women regardless of risk.
Breast density (BD) reflects the amount of glandular and fibrous connective tissue compared with the amount of fatty tissue in the breasts, as seen on a mammogram. BD has three attributes that support use in stratification of population screening. First, increased $\mathrm{BD}$, conditional on age and body mass index (BMI), is a strong risk factor for breast cancer [8]; second, high levels of BD are associated with lower sensitivity of mammography due to masking, i.e., when dense breast parenchyma obscures a cancer and allows it to grow undetected until it is symptomatic $[9,10]$ and third, lower levels of BD are associated with a longer preclinical screen-detectable period [11]. Risk prediction algorithms $[12,13]$ have predominantly used BD as visually assessed by the radiologist according to the Breast Imaging Reporting and Data System (BI-RADS) [14]. Although BI-RADS BD stratifies risk [15], substantial inter-observer variability has generated interest in adopting automated methods [16]. Automated quantitative BD [17-21] would enable more consistent density assessment, and hence, potentially risk assessment for use in breast cancer

${ }^{1}$ Department of Radiology, University of Wisconsin-Madison School of Medicine and Public Health, E3/311 Clinical Science Center, Madison, WI, USA. ${ }^{2}$ National Co-ordinating

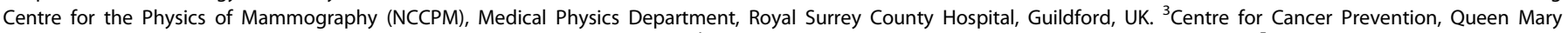

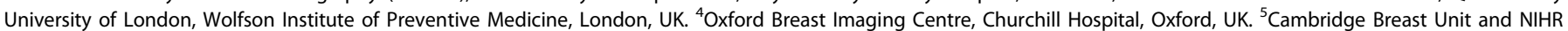

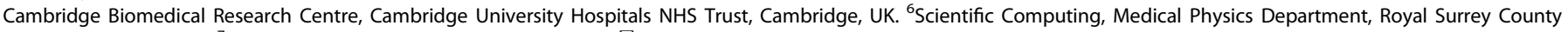
Hospital, Guildford, UK. ${ }^{7}$ American Cancer Society, Atlanta, GA, USA. ${ }^{凶}$ email: eburnside@wisc.edu 
screening protocols. In addition, automated methods may provide the opportunity to disentangle the most predictive components of $\mathrm{BD}$ related to breast cancer risk in a way that visual assessment cannot. For example, quantitative methods can assess whether the absolute or relative amount of BD on mammography (or a combination) represent the key elements that confer breast cancer risk. These algorithms may also be able to separate the risk of breast cancer from the risk of masking, an important distinction when considering the utility of more frequent mammography screening versus the addition of supplemental screening modalities like MRI or ultrasound [22]. Furthermore, few studies have investigated the ability of quantitative $\mathrm{BD}$ analysis to predict the risk of interval [23] or advanced cancers [19].

To fill this gap in the literature, we compared women diagnosed with cancer (interval, node-positive, and screen-detected) to disease-free women with respect to BD. We measured BD using automated $\mathrm{BD}$ assessments and radiologists' quantitative visual $\mathrm{BD}$ assessments to compare the predictive ability of each BD assessment method. We hypothesised that quantitative BD can predict interval cancers and node-positive screen-detected cancers in order to serve as an imaging biomarker with the potential to personalise breast cancer screening.

\section{METHODS}

Ethical approval for the establishment and use of the OPTIMAM image database [24] was obtained from the NHS National Research Ethics Service.

\section{Study population}

In the National Health Breast Screening Programme (NHSBSP), women aged 50-70 years are invited for screening every three years, with an age extension being piloted in a randomised controlled trial of women 47-73 years conducted from 2009 to 2022 [25]. We specifically selected women aged 47-73 who underwent mammographic screening between May 2011 and March 2016 at the Jarvis Breast Screening Centre (Guildford, Surrey, UK) and otherwise met the inclusion criteria for the study. The NHSBSP in general, and the Jarvis Breast Screening Centre specifically, started to convert to digital mammography in 2011. We conducted a retrospective case-control study using mammographic screening images and associated pathological data that were collected as part of the research image database called the OPTIMAM Image Database [24]. Because adherence in a screening program is never exactly within the prescribed round length, due to patient or programme factors (e.g. delayed invitations) we allowed interval cancers to include those found between screening, regardless of timing. This definition means interval cancers are equivalent to postscreening symptomatic cancers in our analysis.

The images were acquired on five Hologic Selenia systems, two Hologic Selenia Dimensions systems (Hologic Inc., Bedford, USA), one GE Senographe Essential system (GE Healthcare Inc., Chicago, USA) and one Sectra MDM-L30 (Phillips Healthcare, Cambridge, Massachusetts, USA). All the digital mammograms in the study were de-identified. Both unprocessed and processed images were collected, when available. To be included in the study, women needed at least one negative digital mammogram prior to the screening mammogram that detected their cancer or the diagnostic mammogram that diagnosed their interval cancer. For the screen-detected cancers, the prior mammogram was used in the study in order to provide an assessment, whether by the radiologist or quantitative imaging, that was 'blind' to the cancer. Selection of controls for each case followed a prescribed protocol. Cancer free controls were selected based on the same equipment and 'date of acquisition' as the cases. For screen-detected cases, 'date of acquisition' was the date of screening examination at which time the cancer is detected. For interval cancers, there were no screening images for detection of cancer (by definition), so 'date of acquisition' was date of prior screening images for that individual. From the group of controls meeting these requirements for each case (machine and 'date of acquisition'), the closest available age was selected. This resulted in $99.4 \%$ of cases and controls being within 4 years of age. Because of the limited normal cases in the OPTIMAM database at the time of case/control selection, a one-to-one match protocol was not possible for all. In total, 542 cases had matched controls and 57 cases did not. Thus 63 unmatched controls were included. Matching on other characteristics (e.g. ethnicity or BMI) was not possible because such variables were not available. All the controls were followed up and remained cancer free for at least 3 years. Pathological data were collected from England's National Breast Screening System.

We required adequate statistical power for comparison of controls with two specific subgroups of cases: interval cancers and node-positive cancers. For both these case groups, we posited that $\sim 20 \%$ of controls and $30 \%$ of cases would be in the highest density category. Estimating that the total number of controls would be at least double the number of cases in either of these subgroups, 291 cases would give $90 \%$ power and 216 cases would give $80 \%$ power. We, therefore, aimed to have at least 216 cases in each subgroup. Anticipating that, for some cases and controls, the unprocessed mammograms might not be available, we obtained 599 cases in total, comprising 302 screen-detected cancers and 297 interval cancers. We sought to enrich the dataset for node-positive cases, so all available node-positive cases $(n=239)$ were selected, and node-negative cases ( $n=$ 360 ) were selected randomly to complete the case set.

\section{Breast density assessment}

Automated BD software (Volpara Health Technologies Ltd: Version 1.5.1, New Zealand) was used to calculate fibroglandular volume (FGV) in $\mathrm{cm}^{3}$, volumetric breast density (VBD) in percent and 5th Edition Volpara Density Grade (DG) from the unprocessed images on the exam level. Volpara is a FDA-approved fully automated software to estimate volumetric breast density [26], based on a detailed relative physics model whereby a region of the breast which is entirely fatty tissue is identified and used as a reference to then calculate the thickness of fibroglandular tissue at each pixel of the image [27]. A model of the breast under compression and the breast thickness (from the DICOM header) are used to convert these fibroglandular tissue thicknesses to volumes, which are then summed across the breast. to provide the FGV and VBD per image. For each screening exam (i.e. a typical four-view exam comprises of the left and right, cranio-caudal (CC) and mediolateral oblique (MLO) views), Volpara software aggregates the image-level metrics to output study-level results per exam. For each breast side, FGV and VBD are averaged across the two views (i.e. CC and MLO), to provide per-breast results for the left and right breasts separately. The study-level FGV and VBD were calculated as the mean of the two per-breast results.

In addition, VBDmax is calculated as the denser VBD of the left or right breasts. Volpara software uses preset cut-off points of VBDmax (to mimic BI-RADS 5th Edition) and reports a study-level 5th Edition Volpara Density Grade (DG), where DG a: $0 \leq \mathrm{VBD}<3.5 \%$, DG b: $3.5 \% \leq \mathrm{VBD}<7.5 \%$, DG c: $7.5 \leq \mathrm{VBD}<15.5 \%$, DG d: VBD $\geq 15.5 \%$ ). Typically, the Volpara Density Grades are denoted as VDG a/b/c/d. However, to avoid confusion between acronyms that designate ' $\mathrm{V}$ ' as 'volume' or 'volumetric' the acronym DG is used throughout this paper, rather than VDG. Volpara software has been validated [26] and used extensively [28] by other groups.

A radiologist (ESB), blinded to case-control status, was shown the images using MedXViewer [29] and assessed BD on a visual analogue scale (VAS) from 0 to 100 for each exam following guidance in prior literature [28].

\section{Statistical analysis}

We took the continuous variables (FGV, VBD and VAS) and determined categorical quartiles using thresholds determined by the distribution for all cases and controls combined (excluding those missing raw images). DG is a categorical variable, already divided by the Volpara software into categories with pre-determined thresholds. We then estimated how these four categorical measures of BD (FGV-quartile, VBD-quartile, VAS-quartile and $D G$ ) and how three continuous BD measures (FGV, VBD and VAS) discriminated between cases and controls. We estimated the effects of these $\mathrm{BD}$ variables on risk of cancer overall and on the risk of particular subsets of cancers (node-positive, node-negative, interval, and screendetected) using logistic regression, adjusting for age. For each subgroup of cases, we used all controls as the comparator group.

In addition, we carried out receiver operating characteristic (ROC) analysis, by estimating and comparing areas under the ROC curve (AUCs). We used the De Long et al. [30] method to compare AUCs between BD measures. We compared AUCs between different cases subgroups using permutation tests [31]. Finally, we also provide a, perhaps, more clinically relevant, measure of discrimination showing the numbers in the lowest risk $25 \%$ ( 1 st quartile) and the highest risk 25\% (4th quartile) of each 'type' of cancer. 
Table 1. Description of the study population and cancer cases.

\begin{tabular}{|c|c|c|c|c|c|c|}
\hline & \multicolumn{2}{|l|}{ Control } & \multicolumn{2}{|c|}{ Screen-detected } & \multicolumn{2}{|c|}{ Interval } \\
\hline & $\#$ & (\%) & $\#$ & (\%) & $\#$ & (\%) \\
\hline Mammograms & $N=605$ & & $N=302$ & & $N=29$ & \\
\hline \multicolumn{7}{|l|}{ Age } \\
\hline 47-49 & 37 & 6.1 & 14 & 4.6 & 22 & 7.4 \\
\hline $50-54$ & 122 & 20.2 & 63 & 20.9 & 68 & 22.9 \\
\hline $55-59$ & 126 & 20.8 & 62 & 20.5 & 62 & 20.9 \\
\hline $60-64$ & 154 & 25.5 & 79 & 26.2 & 63 & 21.2 \\
\hline $65-69$ & 138 & 22.8 & 76 & 25.2 & 64 & 21.5 \\
\hline $70-73$ & 28 & 4.6 & 8 & 2.6 & 18 & 6.1 \\
\hline \multicolumn{7}{|c|}{ Date of 'prior' mammogram } \\
\hline 2010 & 27 & 4.5 & 12 & 4 & 17 & 5.7 \\
\hline 2011 & 84 & 13.9 & 46 & 15.2 & 41 & 13.8 \\
\hline 2012 & 292 & 48.3 & 204 & 67.5 & 81 & 27.3 \\
\hline 2013 & 138 & 22.8 & 38 & 12.6 & 101 & 34 \\
\hline 2014 & 51 & 8.4 & 2 & 0.7 & 45 & 15.2 \\
\hline 2015 & 13 & 2.1 & 0 & 0 & 12 & 4 \\
\hline \multicolumn{7}{|l|}{ Machine } \\
\hline Hologic & 593 & 98.0 & 296 & 98.0 & 288 & 97.0 \\
\hline GE & 9 & 1.5 & 6 & 2.0 & 9 & 3.0 \\
\hline Sectra & 3 & 0.5 & 0 & 0 & 0 & 0 \\
\hline \multicolumn{7}{|l|}{ Invasive/In situ } \\
\hline Invasive & & & 245 & 81.1 & 279 & 93.9 \\
\hline In situ & & & 57 & 18.9 & 18 & 6.1 \\
\hline \multicolumn{7}{|l|}{ Nodal status } \\
\hline Positive & & & 116 & 38.4 & 123 & 41.4 \\
\hline Negative & & & 186 & 61.6 & 174 & 58.6 \\
\hline \multicolumn{7}{|c|}{ Number of nodes positive } \\
\hline None & & & 186 & 61.6 & 174 & 58.6 \\
\hline 1,2 or 3 & & & 101 & 33.4 & 89 & 30 \\
\hline 4 or more & & & 15 & 5 & 34 & 11.4 \\
\hline
\end{tabular}

\section{RESULTS \\ Data description}

Our study included 1204 subjects (599 cancers, 605 controls) in women aged 47-73 years old. Dates of mammograms included in this study ranged from 2010 to 2015 (Table 1). Of note, the mammograms included our study for the screen-detected cancers was the prior mammogram. Thus, for the women (defined by the inclusion criteria) who underwent screening between 2011 and 2016 and had a screen-detected cancer, as mentioned in the 'Methods' section, the prior mammogram was therefore dated earlier than the inclusion criteria range. For the 302 screendetected cancers, the time between prior screening exam and diagnosis, as defined by first positive biopsy, was an average 1067 days (range: 454-1196). For the 297 interval cancers, the time between screening and diagnosis was an average of 656 days (range: 26-1991). As expected, a higher proportion of screendetected than interval cancers were in situ, and a higher proportion of interval cancers were node-positive. The 599 cancers in our study included 524 invasive and 75 cases of ductal carcinoma in situ (DCIS) - for more detail, see Supplementary Information, Supplementary Tables 1 and 2.

\section{Categorical quantitative BD predicting cancer types}

Unprocessed images needed for automated BD measures were available for $429(72 \%)$ cases and $418(69 \%)$ controls. FGV-quartile, VAS-quartile, and DG predicted all cancers versus controls, while VBD-quartile did not (Table 2). The steepest risk gradient for all
Table 2. Association of categorical measures of density with cancer risk (all cancers).

\begin{tabular}{|c|c|c|c|c|c|c|c|}
\hline & \multicolumn{2}{|c|}{ Controls } & \multicolumn{2}{|c|}{ All cancers } & \multirow[b]{2}{*}{ OR } & \multirow[b]{2}{*}{$95 \% \mathrm{Cl}$} & \multirow[b]{2}{*}{$p$-value } \\
\hline & \# & $\%$ & $\#$ & $\%$ & & & \\
\hline \multicolumn{8}{|l|}{ FGV $\left(\mathrm{cm}^{3}\right)^{\mathrm{a}}$} \\
\hline 1st quartile & 137 & 22.6 & 75 & 12.5 & 1 & & $p<0.01$ \\
\hline 2nd quartile & 114 & 18.8 & 98 & 16.4 & 1.6 & $(1.1,2.3)$ & \\
\hline 3rd quartile & 95 & 15.7 & 116 & 19.4 & 2.3 & $(1.5,3.4)$ & \\
\hline 4th quartile & 72 & 11.9 & 140 & 23.4 & 3.7 & $(2.5,5.6)$ & \\
\hline Missing & 187 & 30.9 & 170 & 28.4 & & & \\
\hline \multicolumn{8}{|l|}{ VBD $(\%)^{b}$} \\
\hline 1st quartile & 119 & 19.7 & 100 & 16.7 & 1 & & $p=0.12$ \\
\hline 2nd quartile & 107 & 17.7 & 99 & 16.5 & 1.1 & $(0.7,1.6)$ & \\
\hline 3rd quartile & 101 & 16.7 & 111 & 18.5 & 1.3 & $(0.9,1.9)$ & \\
\hline 4th quartile & 91 & 15.0 & 119 & 19.9 & 1.6 & $(1.0,2.3)$ & \\
\hline Missing & 187 & 30.9 & 170 & 28.4 & & & \\
\hline \multicolumn{8}{|l|}{$\operatorname{VAS}(\%)^{c}$} \\
\hline 1st quartile & 174 & 28.8 & 143 & 23.9 & 1 & & \\
\hline 2nd quartile & 157 & 26 & 137 & 22.9 & 1.1 & $(0.8,1.5)$ & $p=0.04$ \\
\hline 3rd quartile & 132 & 21.8 & 165 & 27.5 & 1.5 & $(1.1,2.1)$ & \\
\hline 4th quartile & 142 & 23.5 & 154 & 25.7 & 1.3 & $(0.9,1.8)$ & \\
\hline \multicolumn{8}{|l|}{ DG } \\
\hline 1 & 27 & 4.5 & 14 & 2.3 & 1 & & $p=0.04$ \\
\hline 2 & 206 & 34.0 & 193 & 32.2 & 1.7 & $(0.9,3.5)$ & \\
\hline 3 & 135 & 22.3 & 151 & 25.2 & 2.1 & $(1.0,4.2)$ & \\
\hline 4 & 50 & 8.3 & 71 & 11.9 & 2.6 & $(1.3,5.7)$ & \\
\hline Missing & 187 & 30.9 & 170 & 28.4 & & & \\
\hline
\end{tabular}

Quartile cut-points.

${ }^{\mathrm{a}} \mathrm{FGV}: 11.70,37.95,51.30,73.35,306.50$.

bVD: $2.4,4.8,6.9,10.9,30.0$.

'VAS: $1.9,29.0,47.0,64.0,96.1$.

cancers was associated with FGV with an odds ratio (OR) for the highest quartile compared to the lowest of 3.7 (95\% Cl 2.5-5.6).

VAS-quartile was not associated with node-positive cancers. In contrast, all categorical automated BD predicted interval cancers and 'node-positive or interval' cancers (henceforth referred to as 'combined' cancers) with statistical significance (Table 3). FGVquartile, VBD-quartile, and DG statistically significantly predicted node-positive cancers. FGV-quartile demonstrated the steepest risk gradient for interval (OR 5.3, Cl 3.1, 9.1, $p<0.01$ ), node-positive (OR 4.7, $\mathrm{Cl} 2.5,9.0, p<0.01$ ) and combined cancers (OR 4.7, $\mathrm{Cl} 2.9$, $7.8, p<0.01)$. All automated $\mathrm{BD}$ measures more consistently predicted interval compared to screen-detected cancers (Fig. 1)for more detail, see Supplementary Information, Supplementary Table 3.

\section{Continuous BD measures predicting cancer types}

For continuous BD measures (FGV, VBD and VAS), the differences in means between cases and controls were statistically significant for all, interval, node-positive, and combined cancers (Table 4). The difference in means for FGV between cases and controls was statistically significant for screen-detected cancers. The difference in means for FGV and the difference in means for VAS between cases and controls were statistically significant for node-negative cancers.

AUC analysis (Fig. 2) demonstrates that FGV reached the highest discriminative ability with an AUC of 0.65 for three subsets of cancers: interval cancers $(95 \% \mathrm{Cl} 0.60,0.70)$, node-positive cancers $(95 \% \mathrm{Cl} 0.59,0.71)$, and combined cancers $(95 \% \mathrm{Cl} 0.60,0.69)$. FGV, VBD and VAS were each able to discriminate all, interval, nodepositive, and combined cancers from controls, as demonstrated by 


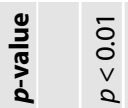

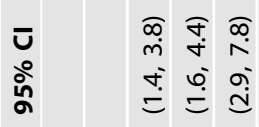

$\stackrel{\infty}{0}-\stackrel{\mathfrak{i}}{\hat{i}} \hat{j}$

$-\mp \stackrel{\infty}{+} \stackrel{m}{i}$

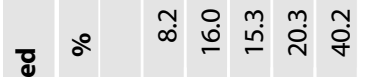

हํํำ

छे \#

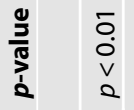

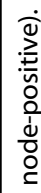

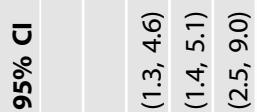

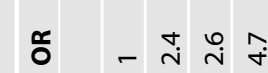

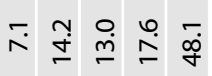

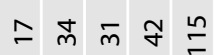

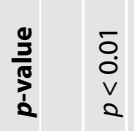

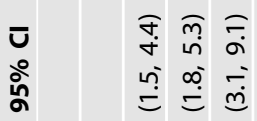

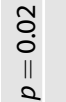

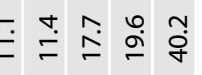

เ $\stackrel{m}{\wedge}$

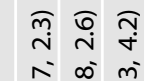

$\hat{\dot{e}} \stackrel{\infty}{\stackrel{\infty}{\stackrel{m}{=}}}$

$-\stackrel{\sim}{\stackrel{4}{*}}$

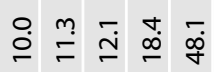

ปั่

5
○’
v
a

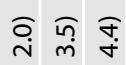

กิ: $\stackrel{m}{=} \stackrel{0}{=}$

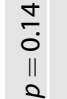

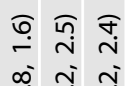

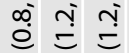

$\overline{0}$
v
Q

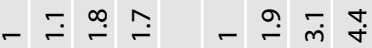

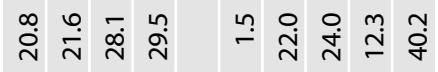

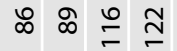

○๐ั

ī.

2

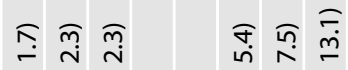

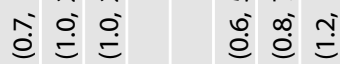

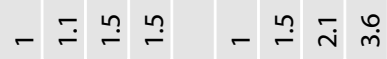

ఇ

命 命

$\bar{c}$
$\dot{0}$
$v$
$a$

$\overline{0}$
0
$v$
2

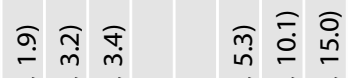

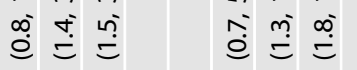

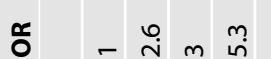

$-\stackrel{ }{\dddot{i}} \hat{i}$

$-\stackrel{-}{4} \frac{4}{4}$

$-\stackrel{m}{\dot{m}} \hat{\dot{\sigma}}$

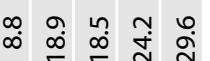

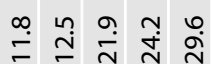

$\stackrel{\infty}{=} \stackrel{\infty}{\circ} \stackrel{\infty}{\infty}$

= ஜூர்

ข้ำ

$\stackrel{m}{m} \hat{m}$ นิำ

นกำ ผ

n

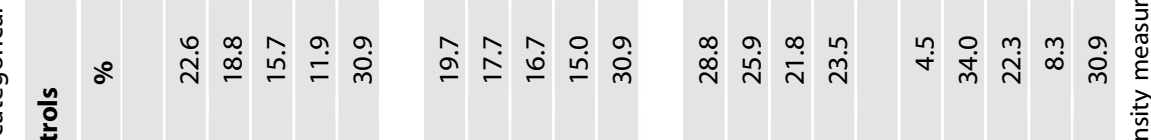

\section{$\stackrel{\circ}{\circ} \doteq \bar{\circ}$}

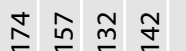

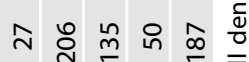

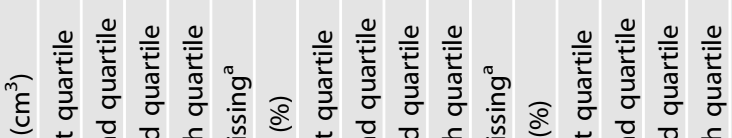

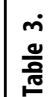

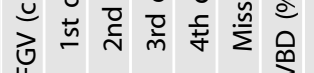

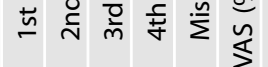




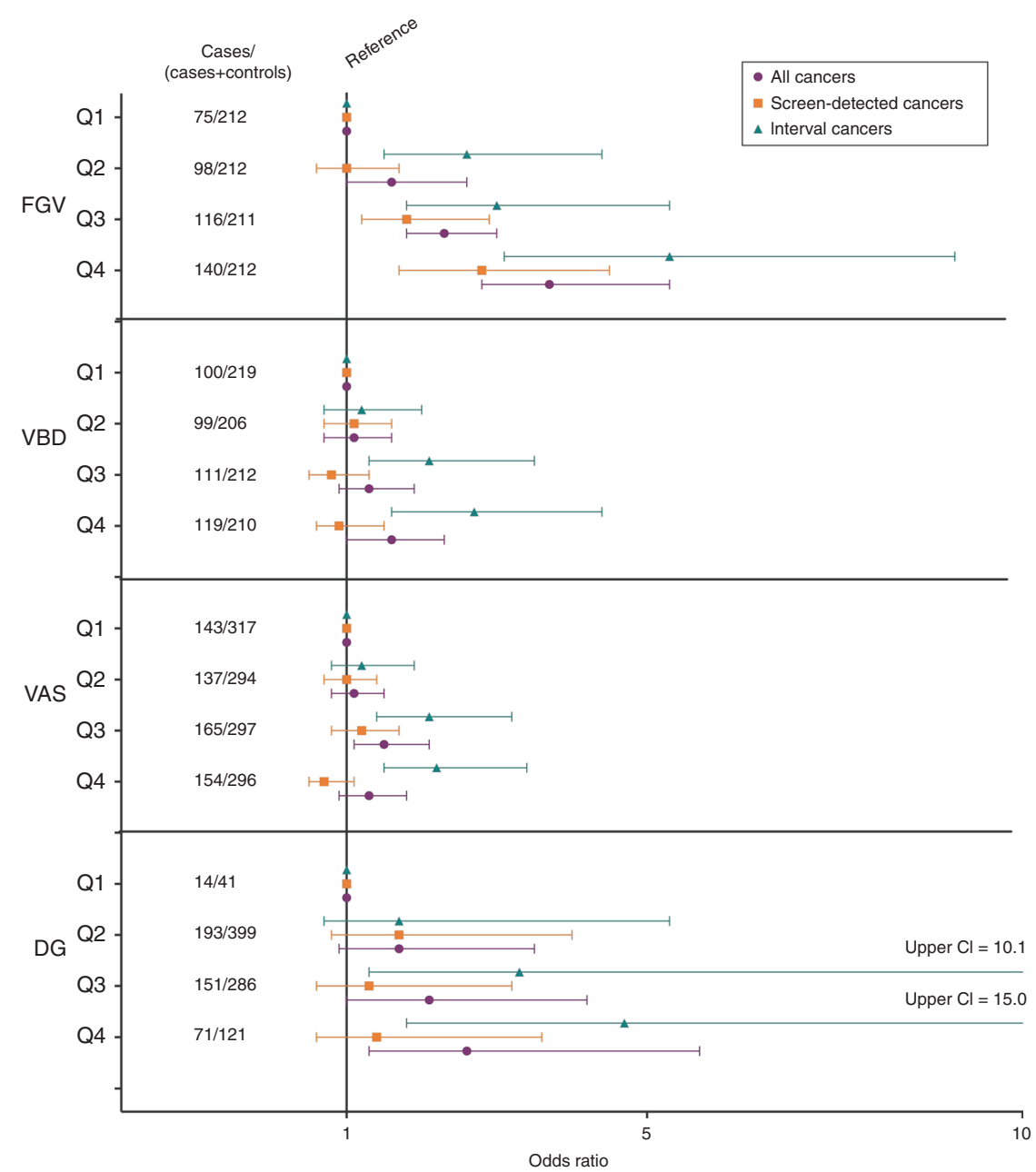

Fig. 1 Visual depiction of BD quartile ability to discriminate all, screen-detected, and interval cancers. Associations between categorical mammographic measures of breast density and breast cancer risk are described by odds ratios for all cancers, screen-detected and interval cancers as compared to controls.

AUC 95\% confidence intervals not including 0.50 (Table 4). VBD and VAS were not able to discriminate screen-detected cancers from controls.

There were clear differences between the AUCs of the three BD measures for all $(p<0.01)$, node-positive $(p<0.01)$ and combined $(p=0.02)$ cancers, but only a moderate difference between BD measures for interval cancers $(p=0.06)$.

To provide a metric that may be more clinically relevant than AUC, we determined the numbers of each 'type' of cancer by risk quartile: the lowest risk $25 \%$ (1st quartile) and the highest risk $25 \%$ (4th quartile). Results showing the highest risk 25\% (4th quartile) for all subcategories of cancers including screen-detected, interval, node-positive, and node-negative demonstrate that FGV captures at least as high a percentage of these cancers as VBD and VAS (Table 5) emulating an exemplar scenario of women who may be candidates for additional screening if the threshold was set below the 4th quartile. FGV categorises at least $10 \%$ more screendetected and node-negative cancers in the highest risk category (4th quartile) as compared VBD and VAS.

FGV, VBD and VAS were all significantly more discriminative of interval cancers than of screen-detected cancers $(p=0.04, p<0.01$ and $p<0.01$ respectively). Only VBD was significantly more predictive of node-positive than of node-negative cancers $(p<$ 0.01 ), although all three measures had greater AUCs for nodepositive than node-negative cancers. All three automated BD methods showed higher AUCs for (i) interval compared to screen- detected; (ii) combined compared to node-negative; and (iii) combined compared to screen-detected cancers. The AUC for interval cancers was significantly greater than the AUC for screendetected cancers for VBD $(p<0.01)$ and VAS $(p<0.01)$, and suggestively so for FGV $(p=0.07)$.

\section{DISCUSSION}

FGV significantly discriminated all, interval, screen-detected, nodepositive and node-negative cancers compared to controls. VBD, VAS and DG discriminated interval or node-positive cancers but did not consistently discriminate screen-detected or nodenegative cancers. The relative discriminative ability of FGV, overall and for each/individual cancer subtypes/groups was either equivalent to or, in most cases, greater than that of VAS or VBD, whether using logistic regression (captured by the steepness of the odds ratio gradient), ROC analysis (captured by AUC), or number of cancers included in the highest risk category (4th quartile). Of note, for VBD and VAS, interval cancer prediction was significantly greater (by AUC) than screen-detected cancer prediction while FGV only showed a statistical trend. This phenomenon underscores the differential ability of FGV to discriminate screen-detected cancers, knowing that FGV has generally higher AUCs for virtually all comparisons (Table 4).

If quantitative breast density is to be successfully used for stratified screening protocols to decrease interval and advanced 
Table 4. Associations of all cancers, screen-detected, interval, node-negative, node-positive and combined (node-positive or interval) cancers with continuous breast density measures.

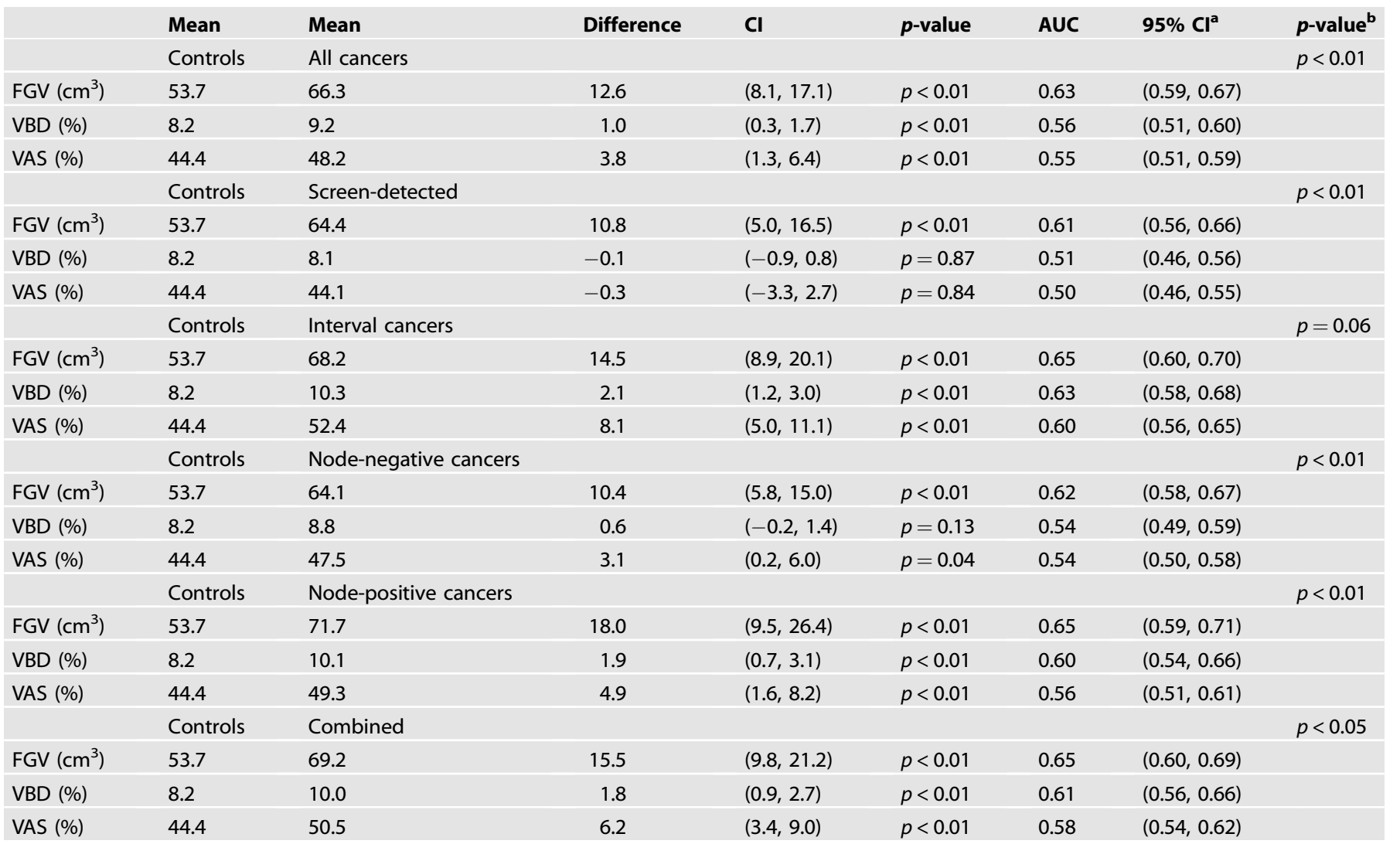

${ }^{\mathrm{a}} 95 \%$ Confidence intervals that do not include 0.50 demonstrate a statistically significantly better discriminatory ability compared to chance.

${ }^{\mathrm{b}}$ This $p$-value reflects whether there is a statistically significant difference between the AUCs of the continuous quantitative BD measurements.

breast cancers, prediction of both the risk of breast cancer and the risk of masking by mammographic breast density will be important. It stands to reason that screen-detected cancers are less affected by masking because they were detected on mammography and, thus, not sufficiently obscured by dense fibroglandular tissue to preclude detection. On the other hand, interval cancers are likely to be more affected by masking because they were not detected by mammography. However, this relationship between interval cancers and masking is far from perfect because interval cancers may also be related to rapid growth between screening examinations or to an interpretation error. Therefore, screen-detected cancers may map more strongly to breast cancer risk as compared to masking. Correspondingly, interval cancers may map more strongly to masking but also involve a component of breast cancer risk. In our study, because VBD and VAS only discriminate interval or node-positive cancers from controls, these algorithms may correlate more strongly with masking. On the other hand, FGV, which additionally discriminates screen-detected cancers from controls may have an added correlation to breast cancer risk. Perhaps FGV maps to both breast cancer and masking risk by measuring absolute BD volume as compared to VBD and VAS, which measure percent BD. There is a precedent for stronger prediction of breast cancer risk generally from absolute rather than percentage density measures [17, 32]. Results, however, are by no means uniform [28]. There is a need for methodological development to disentangle how absolute versus percent fibroglandular volume map to breast cancer risk and masking.

Our results are comparable to results of the single study that analysed interval cancers in a screening programme with a long screening interval (3 years) and tested several quantitative BD techniques [19]. Wanders et al. found that absolute volume of breast density (FGV) predicted screen-detected cancers whereas percent density by volume (VBD) did not [19]. In this study as with the present work, both FGV and VBD predicted interval cancers. Unlike our study, a study by Kerlikowske and colleagues that evaluated the ability of percent density by volume (VBD) to predict screen-detected cancer in a population with a shorter screening interval (1-2 years) showed predictive ability in both screen-detected and interval cancers, with interval cancer prediction being statistically significantly superior [15]. The differences in these results may be attributable to differences in cancers included in screen versus interval groups when the screening interval is shorter, as is the case in the U.S., with a larger fraction of more aggressive cancers included in the interval group. Women in the US may more frequently be offered supplemental screening, influencing patterns of early detection, or be different in terms of breast cancer risk. For example, in the U.S. study, a high proportion (just under 20\%) of controls had a history of breast biopsy [15]. This phenomenon may also relate to the fact that our cases and controls were selected to have the same age distribution, which would remove any density differences between intervals and cancer free controls which were due to confounding with age. Astley et al. [28] found VAS more predictive than the automated measures such as FGV and VBD, but these investigators evaluated images acquired on GE equipment, whereas most images used in our study were acquired on Hologic equipment. The differences in the processed images between these two types of equipment may have affected readers' VAS estimation of BD. The literature shows that image processing significantly affects cancer detection 

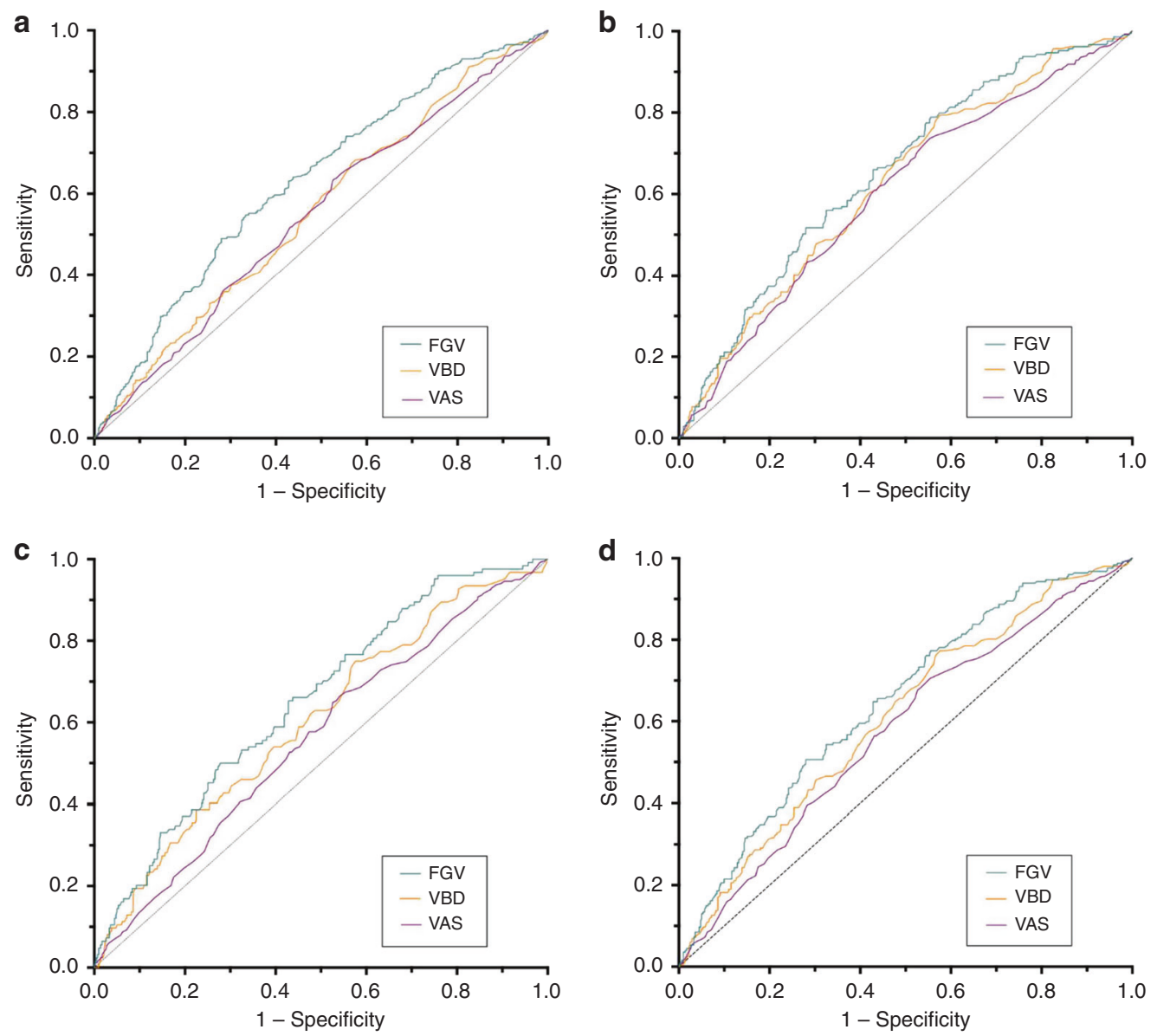

Fig. 2 Ability Continuous BD measures to discriminate cancer types. Receiver operating characteristic (ROC) curves for continuous mammographic measures of breast density to discriminate $\mathbf{a}$ all cancers, $\mathbf{b}$ interval, $\mathbf{c}$ node-positive, and $\mathbf{d}$ combined cancers (interval or nodepositive) from controls.

Table 5. The numbers and percentage of each type of cancer by quantitative density risk quartile.

\begin{tabular}{|c|c|c|c|c|c|c|}
\hline $\begin{array}{l}\text { Density } \\
\text { measure }\end{array}$ & Category & $\begin{array}{l}\text { Controls } \\
N(\%)\end{array}$ & $\begin{array}{l}\text { Screen-detected } \\
\text { cancers } N(\%)\end{array}$ & $\begin{array}{l}\text { Interval cancers } \\
N(\%)\end{array}$ & $\begin{array}{l}\text { Node + Cancers } \\
\text { (\%) }\end{array}$ & $\begin{array}{l}\text { Node-Cancers } \\
\text { (\%) }\end{array}$ \\
\hline \multirow{2}{*}{ FGV } & $\begin{array}{l}\text { 2nd \& 3rd } \\
\text { quartile }\end{array}$ & $209(50)$ & $103(46.8)$ & $111(53.1)$ & $65(52.4)$ & 149 (48.9) \\
\hline & 4th quartile & 72 (17.2) & $68(30.9)$ & $72(34.4)$ & $42(33.9)$ & $98(32.1)$ \\
\hline \multirow[t]{2}{*}{ VBD } & 1st quartile & $119(28.5)$ & $65(29.5)$ & 35 (16.7) & $24(19.4)$ & $76(24.9)$ \\
\hline & 4th quartile & $91(21.8)$ & $47(21.4)$ & $72(34.4)$ & $44(35.5)$ & $75(24.6)$ \\
\hline \multirow[t]{3}{*}{ VAS } & 1st quartile & $174(28.8)$ & $90(29.8)$ & $53(17.8)$ & $55(23)$ & $88(24.4)$ \\
\hline & $\begin{array}{l}\text { 2nd \& 3rd } \\
\text { quartile }\end{array}$ & $289(47.8)$ & $158(52.3)$ & $144(48.5)$ & $118(49.4)$ & $184(51.1)$ \\
\hline & 4th quartile & $142(23.5)$ & 54 (17.9) & $100(33.7)$ & $66(27.6)$ & $88(24.4)$ \\
\hline
\end{tabular}

The highest risk women, 4 th quartile is bolded.

[33], but more work is needed to confirm if image processing also influences visual BD estimation. Overall, the inability to obtain separate estimates for effects on 'pure' masking risk and 'pure' breast cancer risk may account for some of the variation in findings between studies.

Whatever the mechanism for measuring $B D$, women with high levels of BD have an increased risk of interval or node-positive cancers, motivating the need to augment the screening regimen.
Women at high breast cancer risk but not at high masking risk, may benefit from increased mammography screening frequency. Women at high masking risk only or high cancer and masking risk, may be better served by screening with modalities supplementary to mammography, like MRI or ultrasound. In fact, there is interest in determining and targeting these different opportunities for improved screening outcomes (masking versus breast cancer risk) and modelling these strategies [34]. In our study, we find that FGV 
discriminates all categories of cancer more strongly than other density measures perhaps capturing masking and risk more fully than VBD, VAS and DG, which only discriminate interval and nodepositive cancers from controls.

The strengths of our study include our assessment of the discriminative ability of several measures of BD and risk of breast cancer. We also provide an important analysis of volumetric BD related to interval cancer risk $[15,19,35,36]$ and the first related to node-positive cancers. Our cases and controls were selected to have as similar an age distribution as possible, which would remove any density differences between intervals and cancer free controls which were due to confounding by age. However, because density as a risk factor is conditional on age (hence our design and analysis) comparing risks for two women of different ages based on density is not possible based on our work. To fully utilise the risk dimension of density in a screening program, further investigation will be required. For example, a large series of unselected mammograms could be used to construct age-specific reference ranges for density, which would then be a foundation to further refine screening practice.

We did not collect detailed information in relation to a number of covariates (demographic, hormonal, reproductive, lifestyle and family history). We also did not have BMl, which is known to improve discriminatory capacity of quantitative BD measurements [37]. As expected, the time between the analysed mammograms (the most recent normal) for the screen-detected cases was longer than the time between the analysed mammogram and the interval cancers; an unavoidable difference based on the realities of a population-based breast cancer screening programme. This difference raises the question whether adjustment for this difference; i.e. adjustment beyond age may be necessary. We have carried out several major re-analyses incorporating adjustment for time since prior mammogram for those in whom individual matching was possible, revealing no substantive changes to our results or conclusions. Finally, some cases and controls did not have unprocessed images, and thus the quantitative $\mathrm{BD}$ measures were not calculated in these patients. However, our a priori power calculation anticipated these missing images, which therefore should not have influenced our results or conclusions.

We find that FGV has the potential to predict the important components of risk that may provide the foundation for stratified screening: risk of cancer, risk of aggressive cancer, and risk of masking effects. While any quantitative BD measure will undoubtedly be one variable among many predictive variables that will contribute to decisions about breast cancer screening, we believe that our analysis adds to the literature that will inform a more comprehensive model to be tested in the future. Our findings suggest that FGV may be a comparatively better imaging biomarker suited to provide guidance for more intensive stratified screening for mammography, such as a shortened screening interval. VBD, VAS and DG, by predominantly predicting interval cancers and node-positive cancers may selectively correlate with masking risk and be more suited to directing women to supplemental screening modalities other than mammography.

\section{DATA AVAILABILITY}

Mammographic screening images and associated pathological data that were collected as part of the research image database called the OPTIMAM Mammography Image Database cited in the text of the manuscript methods section. The OPTIMAM Mammography Image Database, funded by Cancer Research UK, used in the current study are available and can be found here https://medphys.royalsurrey.nhs.uk/ omidb/.

\section{REFERENCES}

1. Pashayan N, Pharoah P, Tabár L, Neal DE, Martin RM, Donovan J, et al. Validation of a modelling approach for estimating the likely effectiveness of cancer screening using cancer data on prevalence screening and incidence. Cancer Epidemiol. 2011;35:139-44.

2. Evans DG, Howell A. Can the breast screening appointment be used to provide risk assessment and prevention advice? Breast Cancer Res. 2015;17:84.

3. Shieh Y, Eklund M, Madlensky L, Sawyer SD, Thompson CK, Stover Fiscalini A, et al. Breast cancer screening in the precision medicine era: risk-based screening in a population-based trial. J Natl Cancer Inst. 2017;109:1-8.

4. Kerlikowske K, O'Kane ME, Esserman L. Fifty years of age-based screening: time for a new risk-based screening approach. Evid Based Med. 2014;19:183.

5. Evans DG, Brentnall AR, Harvie M, Dawe S, Sergeant JC, Stavrinos P, et al. Breast cancer risk in young women in the national breast screening programme: implications for applying NICE guidelines for additional screening and chemoprevention. Cancer Prev Res. 2014;7:993-1001.

6. Yen AM, Tsau HS, Fann JC, Chen SL, Chiu SY, Lee YC, et al. Population-based breast cancer screening with risk-based and universal mammography screening compared with clinical breast examination: a propensity score analysis of 1429890 Taiwanese women. JAMA Oncol. 2016;2:915-21.

7. Burnside ES, Trentham-Dietz A, Shafer CM, Hampton JM, Alagoz O, Cox JR, et al. Age-based versus risk-based mammography screening in women 40-49 years old: a cross-sectional study. Radiology 2019;292:321-28.

8. McCormack VA, dos Santos Silva I.Breast density and parenchymal patterns as markers of breast cancer risk: a meta-analysis. Cancer Epidemiol Biomarkers Prev. 2006;15:1159-69.

9. Assi V, Warwick J, Cuzick J, Duffy SW. Clinical and epidemiological issues in mammographic density. Nat Rev Clin Oncol. 2011;9:33-40.

10. Chiu SY, Duffy S, Yen AM, Tabar L, Smith RA, Chen HH. Effect of baseline breast density on breast cancer incidence, stage, mortality, and screening parameters: 25-year follow-up of a Swedish mammographic screening. Cancer Epidemiol. Biomarkers Prev. 2010;19:1219-28.

11. Duffy S, Tabar L, Smith R, Krusemo U, Prevost T, Chen T (eds). Risk of breast cancer and risks with breast cancer: the relationship of histologic type with epidemiology, disease progression and survival. Seminars in Breast Disease; 1999.

12. Tice JA, Miglioretti DL, Li CS, Vachon CM, Gard CC, Kerlikowske K. Breast density and benign breast disease: risk assessment to identify women at high risk of breast cancer. J Clin Oncol. 2015;33:3137-43.

13. Brentnall AR, Harkness EF, Astley SM, Donnelly LS, Stavrinos P, Sampson S, et al. Mammographic density adds accuracy to both the Tyrer-Cuzick and Gail breast cancer risk models in a prospective UK screening cohort. Breast Cancer Res. 2015;17:147.

14. American College of Radiology (ACR). Breast Imaging Reporting And Data System (BI-RADS $\left.{ }^{\oplus}\right)$. 5th ed. Reston, VA: ACR; 2014.

15. Kerlikowske K, Scott CG, Mahmoudzadeh AP, Ma L, Winham S, Jensen MR, et al. Automated and clinical breast imaging reporting and data system density measures predict risk for screen-detected and interval cancers: a case-control study. Ann Intern Med. 2018;168:757-65.

16. Conant EF, Sprague BL, Kontos D. Beyond BI-RADS density: a call for quantification in the breast imaging clinic. Radiology. 2018;286:401-4.

17. Duffy SW, Morrish OWE, Allgood PC, Black R, Gillan MGC, Willsher P, et al. Mammographic density and breast cancer risk in breast screening assessment cases and women with a family history of breast cancer. Eur J Cancer. 2018;88:48-56.

18. Wang C, Brentnall AR, Cuzick J, Harkness EF, Evans DG, Astley S. Exploring the prediction performance for breast cancer risk based on volumetric mammographic density at different thresholds. Breast Cancer Res. 2018;20:49.

19. Wanders JOP, Holland K, Karssemeijer N, Peeters PHM, Veldhuis WB, Mann RM, et al. The effect of volumetric breast density on the risk of screen-detected and interval breast cancers: a cohort study. Breast Cancer Res. 2017;19:67.

20. Habel LA, Lipson JA, Achacoso N, Rothstein JH, Yaffe MJ, Liang RY, et al. Casecontrol study of mammographic density and breast cancer risk using processed digital mammograms. Breast Cancer Res. 2016;18:53.

21. Jeffers AM, Sieh W, Lipson JA, Rothstein JH, McGuire V, Whittemore AS, et al Breast cancer risk and mammographic density assessed with Semiautomated and fully automated methods and BI-RADS. Radiology. 2017;282:348-55.

22. Eriksson $M$, Czene $K$, Strand $F$, Zackrisson S, Lindholm $P$, Lång $K$, et al. Identification of women at high risk of breast cancer who need supplemental screening. Radiology. 2020;297:327-33.

23. Boyd NF, Huszti E, Melnichouk O, Martin LJ, Hislop G, Chiarelli A, et al. Mammographic features associated with interval breast cancers in screening programs. Breast Cancer Res. 2014;16:417.

24. Halling-Brown MD, Warren LM, Ward D, Lewis E, Mackenzie A, Wallis MG, et al. OPTIMAM Mammography image database: a large-scale resource of mammography images and clinical data. Radiology: Artificial Intelligence. https://doi.org/ 10.1148/ryai.2020200103.

25. ISRCTN registry. Nationwide cluster- randomised trial of extending the NHS breast screening age range in England [Internet]. http://www.isrctn.com/ ISRCTN33292440. Accessed June 22, 2021. 
26. Gubern-Mérida A, Kallenberg M, Platel B, Mann RM, Martí R, Karssemeijer N. Volumetric breast density estimation from full-field digital mammograms: a validation study. PLoS ONE. 2014;9:e85952.

27. Highnam R, Brady M, Yaffe M, Karssemeijer N, Harvey J, (eds). Robust breast composition measurement-Volpara TM. International Workshop on Digital Mammography; 2010. Berlin, Heidelberg: Springer.

28. Astley SM, Harkness EF, Sergeant JC, Warwick J, Stavrinos P, Warren R, et al. A comparison of five methods of measuring mammographic density: a case-control study. Breast Cancer Res. 2018;20:10.

29. Looney PT, Young KC, Halling-Brown MD. MEDXVIEWER: providing a web-enabled workstation environment for collaborative and remote medical imaging viewing, perception studies and reader training. Radiat Prot Dosimetry. 2016;169:32-7.

30. DeLong ER, DeLong DM, Clarke-Pearson DL. Comparing the areas under two or more correlated receiver operating characteristic curves: a nonparametric approach. Biometrics. 1988;44:837-45.

31. Good P. Permutation tests: a practical guide to resampling methods for testing hypotheses: Springer-Verlag New York; 2013.

32. Assi V, Massat NJ, Thomas S, MacKay J, Warwick J, Kataoka M, et al. A case-control study to assess the impact of mammographic density on breast cancer risk in women aged 40-49 at intermediate familial risk. Int J Cancer. 2015;136:2378-87.

33. Warren LM, Halling-Brown MD, Looney PT, Dance DR, Wallis MG, Given-Wilson $\mathrm{RM}$, et al. Image processing can cause some malignant soft-tissue lesions to be missed in digital mammography images. Clin Radiol. 2017;72:799 e1-e8.

34. Gray E, Donten A, Karssemeijer N, van Gils C, Evans DG, Astley S. et al.Evaluation of a stratified national breast screening program in the United Kingdom: an early model-based cost-effectiveness analysis.Value Health. 2017;20:1100-9.

35. Park IH, Ko K, Joo J, Park B, Jung SY, Lee S, et al. High volumetric breast density predicts risk for breast cancer in postmenopausal, but not premenopausal, Korean Women. Ann. Surgical Oncol. 2014;21:4124-32.

36. Puliti D, Zappa M, Giorgi Rossi P, Pierpaoli E, Manneschi G, Ambrogetti D, et al. Volumetric breast density and risk of advanced cancers after a negative screening episode: a cohort study. Breast Cancer Res. 2018;20:95.

37. Keller BM, Chen J, Daye D, Conant EF, Kontos D. Preliminary evaluation of the publicly available Laboratory for Breast Radiodensity Assessment (LIBRA) software tool: comparison of fully automated area and volumetric density measures in a case-control study with digital mammography. Breast Cancer Res. 2015;17:117.

\section{ACKNOWLEDGEMENTS}

The authors thank Volpara Solutions (Volpara Health Technologies Ltd., New Zealand) for providing Volpara Data Manager software (Version 1.5.1) for this work. This study was performed in accordance with the Declaration of Helsinki.

\section{AUTHOR CONTRIBUTIONS}

Elizabeth Burnside: Conceptualisation, formal analysis, investigation, methodology, project administration, resources, supervision, visualisation, writing-original draft, writing - review and editing. Lucy Warren: Data curation, formal analysis, methodology, software, validation, writing-original draft. Jonathan Myles: Formal analysis, methodology, software, validation. Louise Wilkinson: Conceptualisation, data curation, investigation, resources, visualisation, writing-review and editing. Kenneth Young: Conceptualisation, data curation, funding acquisition, investigation, resources, software, supervision, visualisation, writing - review and editing. Robert Smith: Conceptualisation, funding acquisition, resources, visualisation, writing-review and editing. Nathalie Massat: Conceptualisation, funding acquisition, visualisation, writing-review and editing. Matthew Wallis: Conceptualisation, visualisation, writing-review and editing. Mishal Patel: Conceptualisation, data curation, software, visualisation, writing-review and editing. Stephen Duffy: Conceptualisation, formal analysis, funding acquisition, investigation, methodology, project administration, resources, software, supervision, visualisation, writing —original draft, writing—review and editing.

\section{FUNDING}

The fieldwork for this study was funded by the American Cancer Society (Grant Reference NHPDCSGBR-GBRLONG) and the National Institutes of Health (K24CA194251). This work was part funded by Cancer Research UK, as part of the OPTIMAM2 research programme (Grant Reference C30682/A17321). Stephen Duffy, Nathalie Massat and Jonathan Myles contributed to this work as part of the programme of the Policy Research Unit in Cancer Awareness, Screening and early Diagnosis, PR-PRU-1217-21601, which is funded by the National Institute for Health Research (NIHR) Policy Research Programme. Dr. Wallis was supported by the NIHR Cambridge Biomedical Research Centre (BRC-1215-20014). The views expressed are those of the author(s) and not necessarily those of the NIHR, the Department of Health and Social Care, its arm's length bodies, or other Government Departments. The sponsors of this research did not have a role in the study design, data collection, analysis, the interpretation of the data, or writing of this manuscript. Mishal Patel, is an employee of AstraZeneca. The views expressed are those of the authors and not necessarily those of the AstraZeneca.

\section{ETHICS APPROVAL AND CONSENT TO PARTICIPATE}

Ethical approval for the establishment and use of the OPTIMAM image database was obtained from the NHS National Research Ethics Service. This manuscript does not contain any individual person's data in any form (individual details, images or videos), therefore, no written consent for publication was necessary.

\section{Competing interests}

The authors declare no competing interests.

\section{CONSENT TO PUBLISH}

Not applicable.

\section{ADDITIONAL INFORMATION}

Supplementary information The online version contains supplementary material available at https://doi.org/10.1038/s41416-021-01466-y.

Correspondence and requests for materials should be addressed to E.S.B.

Reprints and permission information is available at http://www.nature.com/ reprints

Publisher's note Springer Nature remains neutral with regard to jurisdictional claims in published maps and institutional affiliations.

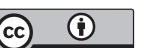

Open Access This article is licensed under a Creative Commons Attribution 4.0 International License, which permits use, sharing, adaptation, distribution and reproduction in any medium or format, as long as you give appropriate credit to the original author(s) and the source, provide a link to the Creative Commons license, and indicate if changes were made. The images or other third party material in this article are included in the article's Creative Commons license, unless indicated otherwise in a credit line to the material. If material is not included in the article's Creative Commons license and your intended use is not permitted by statutory regulation or exceeds the permitted use, you will need to obtain permission directly from the copyright holder. To view a copy of this license, visit http://creativecommons. org/licenses/by/4.0/.

(c) The Author(s) 2021 